EXTENDED REPORT

\title{
Prevalence and associations of an abnormal ankle-brachial index in systemic lupus erythematosus: a pilot study
}

\author{
A Theodoridou, L Bento, D P D'Cruz, M A Khamashta, G R V Hughes
}

Ann Rheum Dis 2003;62:1199-1203. doi: 10.1136/ard.2002.001164

See end of article for authors' affiliations

Correspondence to: Dr D D'Cruz, Lupus Research Unit, The Rayne Institute, St Thomas'

Hospital, London SEl 7EH, UK; david.d'cruz@ kcl.ac.uk

Accepted 5 March 2003
Background: Accelerated atheroma is a well recognised complication of systemic lupus erythematosus (SLE). Its aetiology is multifactorial and several methods may be used to detect early signs of atheroma. Methods: Patients aged $\leqslant 55$ years were screened using the ankle-brachial index (ABI). Ninety one patients aged $\leqslant 55$ years and fulfilling the revised American College of Rheumatology criteria for SLE were studied. The $A B I$ was measured using a contour wrapped $12 \mathrm{~cm}$ cuff attached to a mercury sphygmomanometer and an $8 \mathrm{MHz}$ Doppler probe in the arms and legs; a ratio of $<1$ was considered abnormal.

Results: The mean (SD) age of the patients was 39.0 (9.2) years. Of the 91 patients studied, 34 (37\%) had an abnormal $A B I$. Only one patient was mildly symptomatic. Abnormal $A B I$ correlated with age but not with disease duration, cumulative steroid dosage, ECLAM score, or any other traditional risk factors for atherosclerosis. In comparison with population studies, the prevalence of an $A B I<1$ in the patients with SLE with a mean age of 39 years was similar to that in adults aged over 80 .

Conclusion: In this pilot study, patients with SLE with a mean age of 39 years had a high prevalence of an abnormal $A B I$. The $A B I$ is a simple non-invasive tool for the early detection of accelerated atheroma in SLE.
T he observation that premature atherosclerosis may complicate systemic lupus erythematosus (SLE) was first made by Urowitz and Gladman at the University of Toronto Lupus Clinic. ${ }^{1}$ His team observed a bimodal mortality pattern which occurred in the post-steroid era- "early" deaths due to uncontrolled disease activity and infections and "late" mortality due to atherosclerotic complications, including myocardial infarctions (MIs) and strokes (mean disease duration 8.6 years). They found a fivefold increase in the incidence of MI among lupus patients who were relatively young. There is a substantial increase in coronary heart disease (CHD) and stroke in SLE that cannot be fully explained by the traditional Framingham risk factors alone, ${ }^{2-16}$ even though these risk factors are more common in patients with SLE owing to prednisolone use. SLE has many similarities with the accelerated atherosclerosis seen in diabetes mellitus in that the vascular complications appear early in the disease course. It seems likely that the aetiopathogenesis of cardiovascular disease in this population will be immunological and inflammatory in nature.

There has been considerable interest in this issue and there is a consensus that accelerated atheroma accounts for significant premature mortality rates among lupus patients. ${ }^{1}{ }^{2}$ 4-16 Several recent studies have observed increased mortality rates due to atherosclerosis. These studies include the incidence/prevalence of non-fatal MIs or symptomatic angina and peripheral vascular disease. In contrast, more recent epidemiological studies assessed clinical events such as MI and cardiac death as end points, which almost certainly underestimate the problem. Several studies have also attempted to assess the prevalence of subclinical atheroma in SLE cohorts. Most of these studies used the presence of plaque or intima-medial thickening on B mode carotid ultrasound as the "gold standard".

In addition, there is a need in patients with SLE for better surrogate markers of atherosclerosis than the traditional Framingham risk factors used in population studies. ${ }^{8}$ These may be biological markers, such as $\mathrm{Lp}(\mathrm{a})$ lipoprotein, oxidised low density lipoprotein, homocysteine, heat shock proteins, circulating adhesion molecules, $\beta_{2}$-glycoprotein I, and new autoantibodies. ${ }^{12}{ }^{16}$ Surrogates may also include imaging and functional studies (table 1). The appropriate surrogate should ideally be specific, sensitive, reproducible, preferably non-invasive, affordable, and convenient for screening large numbers of patients with SLE.

In this pilot study, we used the ankle-brachial index (ABI) as a non-invasive screening method to assess the risk of atherosclerotic disease in patients with SLE. The ABI is the ratio of systolic blood pressure at the ankle to the systolic blood pressure at the arm and in a normal healthy person is $\geqslant 1.00$. It is a well established reproducible method with high sensitivity and specificity to assess the patency of the lower limb arterial tree and to detect the presence of peripheral arterial disease. ${ }^{17-40}$ In addition, the ABI has been shown to be a strong predictor of subsequent cardiovascular events in patients with peripheral arterial disease. ${ }^{20} 27$ Several population studies have reported that ABI is inversely associated with mortality or morbidity from atherosclerotic cardiovascular diseases, strokes, and overall mortality. ${ }^{19} 2026-283031$ Further studies suggest that it may be a predictor of the extent of coronary atherosclerosis and cardiovascular events, ${ }^{19-21} 26_{-28}^{30-33}$ and ABI is useful as a screening method for atherosclerosis in comparison with standard methods. ${ }^{19} 21$ Most of the population studies that have been conducted using ABI as a screening method had as end points fatal and non-fatal cardiovascular events (MI, stroke, angina, transient ischaemic attack, etc), 1920252630323639 and all found a two- to fivefold increased risk for these events in subjects with a low ABI. Other studies have evaluated the ability of ABI to predict subclinical disease in comparison with other methods such as carotid Doppler scanning for carotid stenosis and wall

\footnotetext{
Abbreviations: $\mathrm{ABI}$, ankle-brachial index; $\mathrm{aCL}$, anticardiolipin antibodies; aPL, antiphospholipid antibodies; CHD, coronary heart disease; LA, lupus anticoagulant; MI, myocardial infarction; SLE, systemic lupus erythematosus
} 
Table 1 Summary of techniques for the detection of subclinical atherosclerosis

\begin{tabular}{|c|c|}
\hline Coronary angiography & $\begin{array}{l}\text { Invasive and relatively insensitive (does not detect minor stenosis due to } \\
\text { unstable plaque) }\end{array}$ \\
\hline Intracoronary ultrasonography & Sensitive and detects plaque. Invasive and not practical for screening ${ }^{3}$ \\
\hline Echocardiography & $\begin{array}{l}\text { Non-invasive but not sensitive. Detects left ventricular hypertrophy, a } \\
\text { strong risk factor for adverse outcome }\end{array}$ \\
\hline Coronary perfusion & $\begin{array}{l}\text { Thallium perfusion studies and dual isotope myocardial perfusion } \\
\text { imaging (DIMPI): relatively insensitive and may underestimate the } \\
\text { prevalence of atherosclerosis }\end{array}$ \\
\hline $\begin{array}{l}\text { Electron beam computed tomography } \\
\text { (EBCT) }\end{array}$ & $\begin{array}{l}\text { Non-invasive and accurate; detects calcified plaque, a marker of future } \\
\text { cardiac events. Useful only in clinical trials; involves radiation }\end{array}$ \\
\hline Magnetic resonance imaging & Limited resolution owing to cardiac motion ${ }^{3} 40$ \\
\hline$B$ mode ultrasound & $\begin{array}{l}\text { Non-invasive, detects subclinical carotid plaque and intima-media wall } \\
\text { thickness. Accurate and reliable but is operator dependent }{ }^{340}\end{array}$ \\
\hline Myocardial SPECT scan & $\begin{array}{l}\text { Performed after treadmill exercise or dipyridamole stress. Not always } \\
\text { concordant with carotid duplex-ascertained plaque }\end{array}$ \\
\hline Transcranial Doppler & $\begin{array}{l}\text { Microembolic signals on transcranial Doppler ultrasonography are } \\
\text { correlated with atherosclerotic disease }\end{array}$ \\
\hline Vascular stiffness & $\begin{array}{l}\text { Aortic pulse wave velocity (PWV) is an early marker of atherosclerotic } \\
\text { risk. Has been used in } \mathrm{SLE}^{3} 40\end{array}$ \\
\hline Endothelial function & $\begin{array}{l}\text { Flow mediated dilatation measures the brachial artery in response to } \\
\text { reactive hyperaemia. }{ }^{31429} \text { Not yet widely used in routine clinical practice }\end{array}$ \\
\hline
\end{tabular}

thickening, ${ }^{27}{ }^{35-38}$ or fundoscopic examination. ${ }^{24}$ Most patients with a low $\mathrm{ABI}$ are asymptomatic ${ }^{35}{ }^{37}$ and a low $\mathrm{ABI}$ suggests that additional risk assessments should be considered. The ability of a low ABI to predict subsequent events may be greatly increased by combining it with other risk factors. ${ }^{19-21} 32$

The conventional cut off point of a pathological ABI is usually 0.9 and is arbitrary because it was originally developed from studies of patients referred for lower limb angiography. A cut off point of $<0.9$ to 1.0 has conventionally been used for $\mathrm{ABI}$ in the past. The sensitivity and specificity of an $\mathrm{ABI}<1.0$ for $\mathrm{CHD}$ is $41 \%$ and $73 \%$, respectively, and of an $\mathrm{ABI}<0.817 \%$ and $94 \%$, respectively. ${ }^{32}$ A study from Japan calculated the sensitivity and specificity of the various cut off levels of ABI for atherosclerosis, ST segment depression, and ischaemic heart disease and concluded that an $\mathrm{ABI} \leqslant 1.0$ gives the maximum sensitivity and specificity. ${ }^{24} \mathrm{~A}$ recent consensus statement recommends a standard methodology and considers an ABI value $<1.00$ abnormal. ${ }^{41}$

\section{PATIENTS AND METHODS \\ Patients}

Ninety one consecutive patients aged $\leqslant 55$ years and fulfilling the revised American College of Rheumatology classification criteria for SLE $^{41}$ were recruited from our SLE clinics. The Louise Coote Lupus Unit is a tertiary referral centre, and the patients there are representative of lupus patients from all over Great Britain and are in general towards the more severe end of the spectrum of SLE.

Approval for the study was obtained from the St Thomas' Hospital ethics committee on medical research.

\section{Non-invasive vascular assessment}

The ankle-brachial index determination was performed by a single observer (AT) on all subjects after a five minute rest in a supine position. The systolic pressure in the brachial, radial, posterior tibial, and dorsalis pedis arteries in all limbs was measured with a contour wrapped $12 \mathrm{~cm}$ cuff attached to a mercury sphygmomanometer and an $8 \mathrm{MHz}$ Doppler probe (MD 2000). The cuff was inflated to $20 \mathrm{~mm} \mathrm{Hg}$ above the audible systolic pressure in each artery. The recorded systolic pressure was the pressure at which the Doppler probe sounds were first audible as the cuff was slowly deflated. The order of measurement in each limb was the same for all participants. To calculate the $\mathrm{ABI}^{44}$ we used the highest brachial pressure between the two arms. Where the brachial pressure could not be measured, we used the radial pressure instead. To obtain an ankle pressure-for example, on the left, we selected the higher of the left posterior tibial and left dorsalis pedis values. We then divided the highest brachial pressure with the selected ankle pressure to obtain the ABI value for that side. We then used the lower ABI value of the left and right limbs. ${ }^{18} 19223133-35$ We excluded participants with an $\mathrm{ABI} \geqslant 1.5$ because previous studies ${ }^{27} 3238$ suggest that this is a falsely high level caused by non-compressible calcified vessels in the legs. We considered an ABI of $<1.00$ as abnormal as recommended by the consensus statement. ${ }^{44}$

\section{Demographic and other data}

All patients were screened clinically for conventional atherosclerotic risk factors (weight, height, smoking status, family history of cardiovascular disease) and by notes review (diabetes, nephrotic syndrome, history of thrombosis, presence of Raynaud's phenomenon, current or past steroid treatment). We calculated the European Consensus Lupus Activity Measurement (ECLAM) score $^{42}$ and took blood to determine random blood cholesterol, triglyceride and glucose concentrations, thyroid function test status, and lupus serology (anti-DNA, complement, antiphospholipid antibodies (aPL), including anticardiolipin antibodies (aCL) and the lupus anticoagulant (LA)), on the day of the ABI measurement. We also reviewed the files for the presence of at least one previous positive testing of aPL (aCL and LA) and for the presence of antiphospholipid syndrome according to the Sapporo classification criteria. ${ }^{43}$

\section{Statistical analysis}

Univariate associations between demographic, atherosclerotic risk factors, and abnormal ABI ratios were analysed using $\chi^{2}$ test and Student's $t$ test when appropriate. Multivariate analysis was performed with logistic regression for the presence of pathological ABI. All analyses were performed using the NCSS statistical software. A p value $<0.05$ (two tailed) was considered significant.

\section{RESULTS}

Thirty four of the 91 patients studied (37\%) had an abnormal $\mathrm{ABI}(\mathrm{ABI}<1)$ and the other $57(63 \%)$ had normal ABI values $\geqslant 1$. Only one patient aged 26 had mild calf pain on walking $500 \mathrm{~m}$ and none of the others had symptoms or signs of peripheral vascular disease. The ABI values (mean value and standard deviation) were 1.015 (0.126), 1.097 (0.082), and 


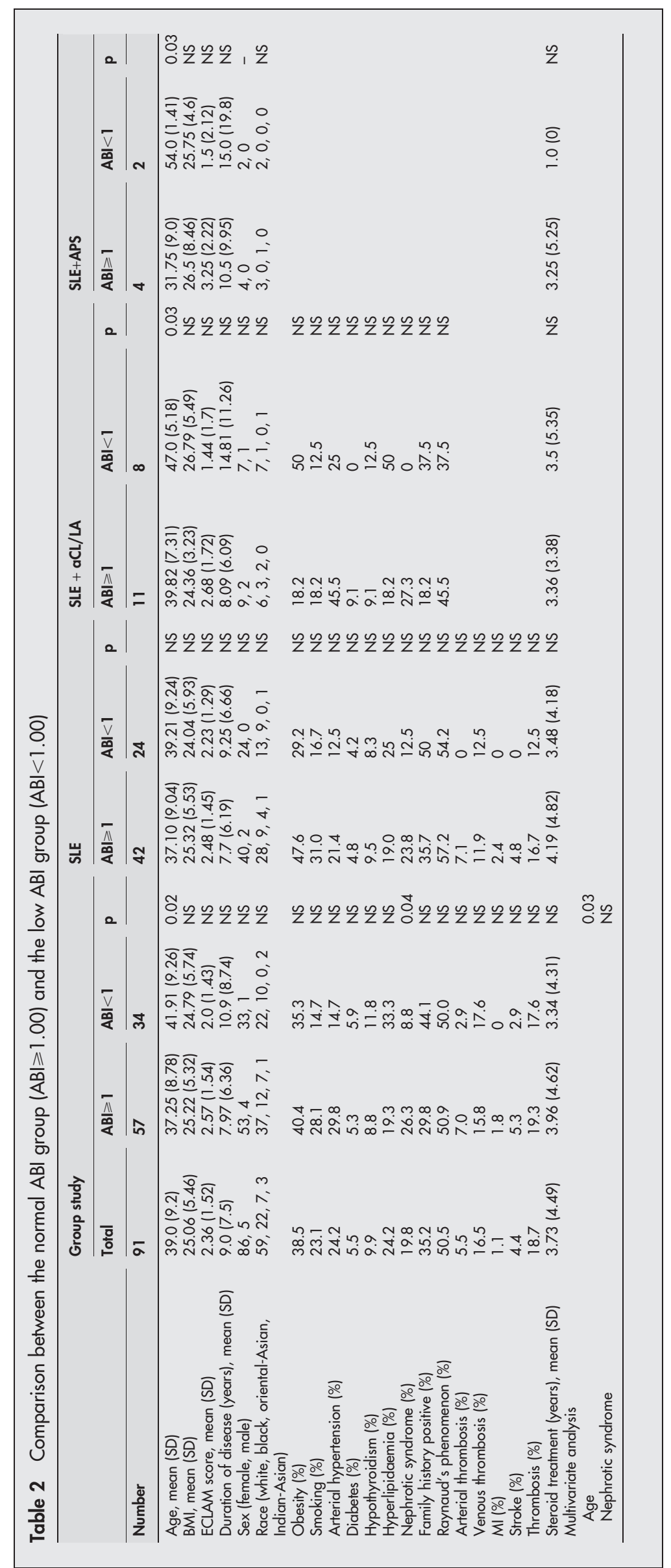


$0.89(0.08)$ for the whole group $(n=91)$, the group with normal ABI $(n=57)$, and the group with low ABI $(n=34)$, respectively. Fifty nine patients (65\%) were white, $22(24 \%)$ were black, $7(8 \%)$ were oriental-Asian, and $3(3 \%)$ were Indian-Asian. In the normal ABI group the distribution was: $37(65 \%), 12(21 \%), 7(12 \%)$, and $1(2 \%)$, respectively, and in the abnormal ABI group the distribution was: 22 (65\%), 10 $(29 \%), 0$, and $2(6 \%)$, respectively. No statistical differences were found between the 86 female patients and the five men. Five patients had antiphospholipid syndrome, of whom two had an abnormal ABI. No significant differences were found between the normal and the abnormal ABI groups for the various risk factors and other variables, except in the age of the patients.

We divided the patients with SLE into two groups according to the presence or absence of aCL/LA or the presence of the antiphospholipid syndrome. We found no difference between the normal/abnormal groups except in the age of the patients. Table 2 shows these results.

\section{DISCUSSION}

It is estimated that mortality due to coronary artery disease accounts for up to $30 \%$ of all deaths in patients with SLE. ${ }^{1-7}$ The prevalence of coronary artery disease is estimated to be $8.3-15 \%$, with a mean age of onset of coronary artery disease of 47.5 years. ${ }^{2}$ The female lupus patients from the Pittsburgh SLE cohort in the 35-44 age group were over 50 times more likely to have an MI than controls. ${ }^{4}$ According to Ward's study, the risk of admission to hospital owing to a cerebrovascular accident was 2.03 times greater for lupus patients aged 18-44 years, ${ }^{7}$ and according to a Canadian study, the overall risk for MI conferred by SLE after controlling for the Framingham risk factors was increased by an estimated 8.3 -fold. ${ }^{8}$ The true prevalence of subclinical disease is not yet certain but there is evidence that markers of subclinical disease may be able to identify subjects at high risk of clinical CHD and those most likely to benefit from aggressive treatment. ${ }^{29}{ }^{40}$

There has been a dramatic change in the approach to studying cardiovascular disease. Table 1 summarises the techniques used for the detection of subclinical atherosclerosis. The current "gold standard" non-invasive method for detecting subclinical atherosclerosis is B mode carotid ultrasound to assess plaque and intima-media thickness.

The ABI measurement can be conducted at low cost, using simple techniques and is non-invasive and not as operatordependent as carotid ultrasound. It is an easy, quick, accurate, and reproducible measurement using a portable probe and sphygmomanometer that is relatively inexpensive. Furthermore, only minimal training is required to use the technique. It can be used as a primary prevention tool in routine screening of cardiovascular status in the community 20 24-27 30323637 and has high patient acceptability. Even though the ability of $\mathrm{ABI}$ to predict subsequent events is increased by combining a low ABI $(<1.00)$ with other risk factors,$^{20}$ its association with cardiovascular events is strongly independent of these other risk factors. ${ }^{25-27}$ The ability of ABI to predict cardiovascular events is increased in those subjects with no clinical cardiovascular disease. ${ }^{27}$

Epidemiological studies show that subjects with clinical cardiovascular disease related to one specific vascular bed (for example, intermittent claudication), are at higher risk of clinical disease caused by atherosclerosis (which is a diffuse disease) in other vascular beds. Most subjects with decreased $\mathrm{ABI}$ are asymptomatic and a high percentage of these subjects have other aspects of subclinical or clinical cardiovascular disease..$^{20} 26^{27}$ Thus a decreased ABI is predictive in an individual patient of a high risk of future cardiovascular events. Such asymptomatic patients are amenable to further investigations and interventional treatments. In asymptomatic disease, aspirin may be as effective as in symptomatic disease, because the cardiovascular disease rate in asymptomatic subjects with a low ABI, is similar to those with clinical disease. ${ }^{21} 262838$

In attempting to prevent initial cardiovascular events for patients with SLE, a reasonable strategy would be to target and treat patients with asymptomatic subclinical atherosclerosis. It might then be possible to reduce their risk with additional monitoring and careful control of risk factors, such as hyperlipidaemia, considering antiplatelet drugs, and encouraging lifestyle changes such as stopping smoking, diet, and exercise. Several studies suggest that SLE itself is a risk factor for atheroma and may result from vascular inflammation mediated by immune complexes. Thus control of disease activity may also help to reduce the burden of atherosclerotic disease.

Measurement of the ABI can identify subjects at high risk, but in general cannot be used to exclude subjects believed not to be at risk of cardiovascular disease. ${ }^{28}$ In other words, a normal ABI does not rule out the presence of atherosclerosis. Although sensitivity is low, it does not differ appreciably from the sensitivity of more common risk factors for cardiovascular disease mortality. In addition, the magnitude of risk for cardiovascular disease that is associated with a low ABI is similar to findings from more extensive testing, including echocardiography and carotid ultrasound. ${ }^{19} 26-28303638$

We studied 91 patients with SLE for traditional risk factors for atherosclerosis and we screened them with an ABI for possible subclinical atherosclerosis. Using a cut off point of $<1.00$ for ABI, we found that $37 \%$ of our patients had an abnormal ABI. In population studies of adults aged under 55 the prevalence of an $\mathrm{ABI} \leqslant 0.9$ is below $4 \%,{ }^{2031} 37$ and this percentage increases rapidly with age. In a prospective population study of healthy adults ${ }^{32}$ the highest prevalence of an abnormal ABI (35\%) was in the $80-84$ year old age group. Thus our young patients with SLE had a prevalence of abnormal ABI values comparable with that of a much older age group. Interestingly, no correlation was found between a low $\mathrm{ABI}$ and the prevalence of aPL, which are a strong predictor of arterial and venous cardiovascular events. Again, this may be a function of the small study numbers but may also be because aPL and the ABI are markers for different vascular processes.

In contrast with other studies, ${ }^{19} 2023-2830323537$ our study failed to show a statistically significant correlation between low ABI and common risk factors. This may be due to $(a)$ the small number of subjects in this pilot study; $(b)$ the fact that the ABI is an independent predictor of mortality, as shown by previous studies. ${ }^{19} 2036$ There is also a paradox in our results relating to steroid treatment; this is of slightly shorter duration in the group with a low $\mathrm{ABI}$ which has longer disease duration than in the group with normal ABI. This is not of statistical significance and may reflect the small number of subjects, or the possibility that the immune-inflammatory nature of the disease influences this phenomenon of early atherosclerosis independently of the traditional risk factors ${ }^{45-12}$ and, according to Manzi et al, ${ }^{4}$ may be stronger than traditional factors.

In conclusion, the $\mathrm{ABI}$ is a simple, non-invasive technique for the detection of accelerated atheroma in young patients with SLE. Our pilot study demonstrates that young patients with SLE have a high prevalence of an abnormal ABI suggesting widespread asymptomatic vascular dysfunction. Clearly, further studies with a control group will help to define the clinical use of this test and how individual patients should be investigated. 


\section{ACKNOWLEDGEMENTS}

We are grateful to the St Thomas' Lupus Trust for the purchase of the Doppler probe.

\section{Authors' affiliations}

A Theodoridou, L Bento, D P D'Cruz, M A Khamashta, G R V Hughes, Lupus Research Unit, The Rayne Institute, St Thomas' Hospital, London, UK

\section{REFERENCES}

1 Urowitz MB, Gladman DD. Accelerated atheroma in lupus-background. Lupus 2000;9:161-5.

2 Aranow C, Ginzler EM. Epidemiology of cardiovascular disease in systemic lupus erythematosus. Lupus 2000;9:166-9.

3 Manzi S, Kuller LH, Edmundowicz D, Sutton-Tyrrell K. Vascular imaging: changing the face of cardiovascular research. Lupus 2000;9:176-82

4 Manzi S, Meilahn EN, Rairie JE, Conte CG, Medsger TA Jr, JansenMcWilliams $L$, et al. Age-specific incidence rates of myocardial infarction and angina in women with systemic lupus erythematosus: comparison with the Framingham Study. Am J Epidemiol 1997;145:408-15.

5 Ward MM, Pyun E, Studenski S. Causes of death in systemic lupus erythematosus. Long-term follow-up of an inception cohort. Arthritis Rheum 1995;38:1492-9

6 Jacobsen S, Petersen J, Ullman S, Junker P, Voss A, Rasmussen JM, et al. A multicentre study of 513 Danish patients with systemic lupus erythematosus. II. Disease mortality and clinical factors of prognostic value. Clin Rheumatol 1998; 17:478-84.

7 Ward MM. Premature morbidity from cardiovascular and cerebrovascular disease in women with systemic lupus erythematosus. Arthritis Rheum 1999:42:338-46.

8 Esdaile JM, Abrahamowicz M, Grodzicky T, Li Y, Panaritis C, du Berger R, et al. Traditional Framingham risk factors fail to fully account for accelerated atherosclerosis in systemic lupus erythematosus. Arthritis Rheum 2001:44:2331-7.

9 Petri M. Detection of coronary artery disease and the role of traditional risk factors in the Hopkins lupus cohort. Lupus 2000;9:170-5.

10 Manzi S. Systemic lupus erythematosus: a model for atherogenesis? Rheumatology (Oxford) 2000;39:353-9.

11 Roman MJ, Salmon JE, Sobel R, Lockshin MD, Sammaritano L, Schwartz JE, et al. Prevalence and relation to risk factors of carotid atherosclerosis and left ventricular hypertrophy in systemic lupus erythematosus and antiphospholipid syndrome. Am J Cardiol 2001;87:663-6.

12 Lockshin MD, Salmon JE, Roman M. Atherosclerosis and lupus: a work in progress. Arthritis Rheum 2001;44:2215-17

13 Selzer F, Sutton-Tyrrell K, Fitzgerald S, Tracy R, Kuller L, Manzi S. Vascular stiffness in women with systemic lupus erythematosus. Hypertension 2001;37:1075-82.

14 Lima DS, Sato El, Lima VC, Miranda F Jr, Hatta FH. Brachial endothelial function is impaired in patients with systemic lupus erythematosus. J Rheumatol 2002;29:292-7.

15 Bruce IN, Burns RJ, Gladman DD, Urowitz MB. Single photon emission computed tomography dual isotope myocardial perfusion imaging in women with systemic lupus erythematosus. I. Prevalence and distribution of abnormalities. J Rheumatol 2000;27:2372-7.

16 llowite NT. Premature atherosclerosis in systemic lupus erythematosus. J Rheumatol 2000;27(suppl 58):15-19.

17 Jeelani NU, Braithwaite BD, Tomlin C, MacSweeney ST. Variation of method for measurement of brachial artery pressure significantly affects anklebrachial pressure index values. Eur J Vasc Endovasc Surg 2000;20:25-8.

18 Calculating the ankle-brachial index. Adv Skin Wound Care 2000;13:86.

19 Papamichael CM, Lekakis JP, Stamatelopoulos KS, Papaioannou TG Alevizaki MK, Cimponeriu AT, et al. Ankle-brachial index as a predictor of the extent of coronary atherosclerosis and cardiovascular events in patients with coronary artery disease. Am J Cardiol 2000;86:615-18.

20 Vogt MT, McKenna M, Wolfson SK, Kuller LH. The relationship between ankle brachial index, other atherosclerotic disease, diabetes, smoking and mortality in older men and women. Atherosclerosis 1993;101:191-202.

21 Leng GC, Fowkes FG, Lee AJ, Dunbar J, Housley E, Ruckley CV. Use of ankle brachial pressure index to predict cardiovascular events and death: a cohort study. BMJ 1996;313:1440-4.
22 McDermott MM Criqui MH, Liu K, Guralnik JM, Greenland P, Martin GJ, et al. Lower ankle/brachial index, as calculated by averaging the dorsalis pedis and posterior tibial arterial pressures, and association with leg functioning in peripheral arterial disease. J Vasc Surg 2000;32:1164-71.

23 Stoffers HE, Kester AD, Kaiser V, Rinkens PE, Kitslaar PJ, Knottnerus JA. The diagnostic value of the measurement of the ankle-brachial systolic pressure index in primary health care. J Clin Epidemiol 1996;49:1401-5.

24 Shinozaki T, Hasegawa T, Yano E. Ankle-arm index as an indicator of atherosclerosis: its application as a screening method. J Clin Epidemiol 1998:51:1263-9.

25 Tsai AW, Folsom AR, Rosamond WD, Jones DW. Ankle-brachial index and 7-year ischemic stroke incidence: the ARIC study. Stroke 2001;32:1721-4.

26 Newman AB, Shemanski L, Manolio TA, Cushman M, Mittelmark M, Polak JF et al. Ankle-arm index as a predictor of cardiovascular disease and mortality in the Cardiovascular Health Study. The Cardiovascular Health Study Group. Arterioscler Thromb Vasc Biol 1999;19:538-45

27 Zheng ZJ, Sharrett AR, Chambless LE, Rosamond WD, Nieto FJ, Sheps DS, et al. Associations of ankle-brachial index with clinical coronary heart disease, stroke and preclinical carotid and popliteal atherosclerosis: the Atherosclerosis Risk in Communities (ARIC) Study. Atherosclerosis 1997;131:115-25

28 Kuller LH. Is ankle-brachial blood pressure measurement of clinical utility for asymptomatic elderly? J Clin Epidemiol 2001;54:971-2.

29 Lima DS, Santo El, Lima VC, Miranda F Jr, Hatta FH. Brachial endothelial function is impaired in patients with SLE. J Rheumatol 2000;29:292-7.

30 Abbott RD, Rodriguez BL, Petrovitch H, Yano K, Schatz IJ, Popper JS, et al. Ankle-brachial blood pressure in elderly men and the risk of stroke: the Honolulu Heart Program. J Clin Epidemiol 2001:54:973-8.

31 Lijmer JG, Hunink MG, van den Dungen JJ, Loonstra J, Smit AJ. ROC analysis of noninvasive tests for peripheral arterial disease. Ultrasound Med Biol 1996;22:391-8.

32 Abbott RD, Petrovitch H, Rodriguez BL, Yano K, Schatz IJ, Popper JS, et al Ankle/brachial blood pressure in men $>70$ years of age and the risk of coronary heart disease. Am J Cardiol 2000;86:280-4.

33 McDermott MM, Mehta S, Liu K, Guralnik JM, Martin GJ, Criqui MH, et al. Leg symptoms, the ankle-brachial index, and walking ability in patients with peripheral arterial disease. J Gen Intern Med 1999;14:173-81.

34 Wan MC, Moore T, Hollis S, Herrick AL. Ankle brachial pressure index in systemic sclerosis: influence of disease subtype and anticentromere antibody. Rheumatology (Oxford) 2001;40:1102-5.

35 Meijer WT, Hoes AW, Rutgers D, Bots ML, Hofman A, Grobbee DE. Peripheral arterial disease in the elderly. The Rotterdam study. Arteriosc Thromb Vasc Biol 1998;18:185-92.

36 McKenna M, Wolfson S, Kuller L. The ratio of ankle and arm arterial pressure as an independent predictor of mortality. Atherosclerosis 1991;87:119-28.

37 Newman AB, Siscovick DS, Manolio TA, Polak J, Fried LP, Borhani NO, et al. Ankle-arm index as a marker of atherosclerosis in the Cardiovascular Health Study. Cardiovascular Heart Study (CHS) Collaborative Research Group. Circulation 1993;88:837-45.

38 Kuller LH, Shemanski L, Psaty BM, Borhani NO, Gardin J, Haan MN, et al. Subclinical disease as an independent risk factor for cardiovascular disease. Circulation 1995;92:720-6.

39 Criqui MH, Langer RD, Fronek A, Feigelson HS, Klauber MR, McCann TJ, et al. Mortality over a period of 10 years in patients with peripheral arterial disease. N Engl J Med 1992;326:381-6.

40 Greenland P, Abrams J, Aurigemma GP, Bond MG, Clark LT, Criqui MH, et al. Prevention conference V: Beyond secondary prevention: identifying the high-risk patient for primary prevention: noninvasive tests of atherosclerotic burden: writing group III. Circulation 2000;101:E16-22.

41 Hochberg MC. Updating the American College of Rheumatology revised criteria for the classification of systemic lupus erythematosus. Arthritis Rheum 1997; $40: 1725$

42 Vitali C, Bencivelli W, Isenberg DA, Smolen JS, Snaith ML, Sciuto M, Hochberg MC, et al. Disease activity in systemic lupus erythematosus: report of the Consensus Study Group of the European Workshop for Rheumatology Research. II. Identification of the variables indicative of disease activity and their use in the development of an activity score. The European Consensus Study Group for Disease Activity in SLE. Clin Exp Rheumatol 1992;10:541-7.

43 Wilson WA Gharavi AE, Koike T, Lockshin MD, Branch DW, Piette JC et al. International consensus statement on preliminary classification criteria fo definite antiphospholipid syndrome: report of an international workshop. Arthritis Rheum 1999;42:1309-11.

44 Sacks D, Bakal C, Beatty P, Becker G, Cardella J, Raabe R, et al. Position statement on the use of the ankle-brachial index in the evaluation of patients with peripheral vascular disease. J Vasc Interv Radiol 2002;13:353. 